\title{
The Evolution of Late\#Time Optical Emission from SN 1986J
}

\section{Citation}

Milisavljevic, Dan, Robert A. Fesen, Bruno Leibundgut, and Robert P. Kirshner. 2008. “The Evolution of Late\#Time Optical Emission from SN 1986J." The Astrophysical Journal 684 (2): 1170-73. https://doi.org/10.1086/590426.

\section{Permanent link}

http://nrs.harvard.edu/urn-3:HUL.InstRepos:41399734

\section{Terms of Use}

This article was downloaded from Harvard University's DASH repository, and is made available under the terms and conditions applicable to Other Posted Material, as set forth at http:// nrs.harvard.edu/urn-3:HUL.InstRepos:dash.current.terms-of-use\#LAA

\section{Share Your Story}

The Harvard community has made this article openly available.

Please share how this access benefits you. Submit a story.

Accessibility 
The Astrophysical Journal, 684:1170-1173, 2008 September 10

(C) 2008. The American Astronomical Society. All rights reserved. Printed in U.S.A.

\title{
THE EVOLUTION OF LATE-TIME OPTICAL EMISSION FROM SN 1986J
}

\author{
Dan Milisavljevic, ${ }^{1}$ Robert A. Fesen, ${ }^{1}$ Bruno Leibundgut, ${ }^{2}$ and Robert P. Kirshner ${ }^{3}$ \\ Received 2008 April 9; accepted 2008 May 23
}

\begin{abstract}
We present late-time optical images and spectra of the Type IIn supernova SN 1986J. HST ACS/WFC images obtained in 2003 February show it to be still relatively bright, with $m_{\mathrm{F} 606 \mathrm{~W}}=21.4$ and $m_{\mathrm{F} 814 \mathrm{~W}}=20.0 \mathrm{mag}$. Compared to 1994 December HST WFPC2 images, SN 1986 J shows a decline of only $\lesssim 1$ mag in brightness over 8 years. Groundbased spectra taken in 1989, 1991, and 2007 show a 50\% decline in $\mathrm{H} \alpha$ emission between 1989 and 1991, and an order of magnitude drop between 1991 and 2007, along with the disappearance of He I line emissions during the period 1991-2007. The object's [O I] $\lambda \lambda 6300,6364$, [O II] $\lambda \lambda 7319,7330$ and [O III] $\lambda \lambda 4959,5007$ emission lines show two prominent peaks near -1000 and $-3500 \mathrm{~km} \mathrm{~s}^{-1}$, with the more blueshifted component declining significantly in strength between 1991 and 2007. The observed spectral evolution suggests two different origins for SN 1986J's late-time optical emission: dense, shock-heated circumstellar material, which gave rise to the initially bright $\mathrm{H} \alpha$, $\mathrm{He}$ I, and [N II] 25755 lines, and reverse-shock-heated O-rich ejecta on the facing expanding hemisphere, dominated by two large clumps generating two blueshifted emission peaks in the [O I], [O II], and [O III] lines.
\end{abstract}

Subject headings: circumstellar matter - supernova remnants - supernovae: general

\section{INTRODUCTION}

With a peak flux density of $128 \mathrm{mJy}$ at $5 \mathrm{GHz}$, SN 1986J is one of the most radio-luminous supernovae ever detected (Weiler et al. 1990). The supernova probably occurred early in 1983 in the edge-on galaxy NGC 891, more than 3 years before its 1986 August discovery in the radio (van Gorkom et al. 1986; Rupen et al. 1987; Bietenholz et al. 2002). With its optical outburst going unnoticed, the earliest optical detection showed the supernova at a magnitude of 18.4 in $R$ in 1984 January (Rupen et al. 1987; Kent \& Schild 1987).

SN 1986J is classified as a Type IIn (see Schlegel 1990) and has been compared with other luminous SNe IIn, such as SN 1988 Z and SN 1995N. Optical spectra of SN 1986J obtained in 1986 showed prominent and narrow $\left(\Delta v \lesssim 700 \mathrm{~km} \mathrm{~s}^{-1}\right) \mathrm{H} \alpha$ emission with no broad component (Rupen et al. 1987). Emission lines of [O I], [O II], and [O III] had somewhat larger widths of $1000<$ $\Delta v<2000 \mathrm{~km} \mathrm{~s}^{-1}$. Many narrow and weak emission lines, including those from helium, were also observed. Spectra taken 3 years later, in 1989, showed that the dominant narrow $\mathrm{H} \alpha$ emission had diminished in strength, with the forbidden oxygen emission lines relatively unchanged (Leibundgut et al. 1991; hereafter L91).

Early very long baseline interferometry (VLBI) revealed an aspherical source with marginal indication of an expanding shell (Bartel et al. 1989, 1991). Subsequent VLBI observations show a distorted shell and a current expansion velocity of $\sim 6000 \mathrm{~km} \mathrm{~s}^{-1}$, considerably less than an extrapolated initial velocity of $\sim 20,000 \mathrm{~km} \mathrm{~s}^{-1}$ (Bietenholz et al. 2002).

Here we present Hubble Space Telescope (HST) images of SN 1986J showing it to still be relatively optically luminous more than two decades after outburst. We also present ground-based optical spectra obtained at three epochs spanning 18 years to follow its late-time emission evolution.

\footnotetext{
1 Department of Physics and Astronomy, 6127 Wilder Lab, Dartmouth College, Hanover, NH 03755.

2 ESO, Karl-Schwarzschild-Strasse 2, Garching, D-85748, Germany.

3 Harvard-Smithsonian Center for Astrophysics, 60 Garden Street, Cambridge, MA 02138
}

\section{OBSERVATIONS}

Images of NGC 891 in the region around SN 1986J obtained by the Advanced Camera for Surveys (ACS) system aboard the HST using the Wide Field Channel (WFC) on 2003 February 18 and 20 were retrieved from STScI archives (GO 9414; PI: R. de Grijs). The images were taken using filters F606W and F814W. Standard IRAF/STSDAS data reduction was done including debiasing, flat-fielding, geometric distortion corrections, photometric calibrations, and cosmic-ray and hot pixel removal, with the STSDAS drizzle task used to combine exposures.

Low-dispersion optical spectra of SN 1986J were obtained with the MMT on Mount Hopkins using the Red Channel spectrograph with a TI $800 \times 800$ CCD on 1989 September 5 and again on 1991 October 14. For both observations, a $2^{\prime \prime}$ wide slit and a 150 lines $\mathrm{mm}^{-1}$ grating was used to obtain spectra spanning $4000-8000 \AA$, with a resolution of $\sim 30 \AA$. Total exposure time for each spectrum was $9000 \mathrm{~s}$.

Spectra of SN 1986J were also obtained on 2007 September 11 at MDM Observatory using the $2.4 \mathrm{~m}$ Hiltner telescope with the Boller \& Chivens CCD spectrograph (CCDS). A north-south $1.5^{\prime \prime} \times 5^{\prime}$ slit and a 150 lines $\mathrm{mm}^{-1} 4700 \AA$ blaze grating was used to obtain two sets of spectra, one consisting of $3 \times 1800 \mathrm{~s}$ exposures spanning 4000-7100 $\AA$, and another of $2 \times 1800 \mathrm{~s}$ exposures covering 6900-9000 $\AA$ using an LG 505 order separation filter. Both spectra were of $\sim 10 \AA$ resolution. Another spectrum with this same setup consisting of $2 \times 3000 \mathrm{~s}$ exposures was taken on 2007 December 20. Seeing was around $1^{\prime \prime}$ for all spectra. The spectra were processed using standard procedures in $\mathrm{IRAF}^{4}$ with standard stars from Strom (1977).

\section{RESULTS}

\subsection{Late-Time Optical Photometry}

The left panel of Figure 1 shows the blue DSS2 image of NGC 891 with the location of SN 1986J marked, and the two right

4 The Image Reduction and Analysis Facility is distributed by the National Optical Astronomy Observatories, which are operated by the Association of Universities for Research in Astronomy, Inc., under cooperative agreement with the National Science Foundation. 


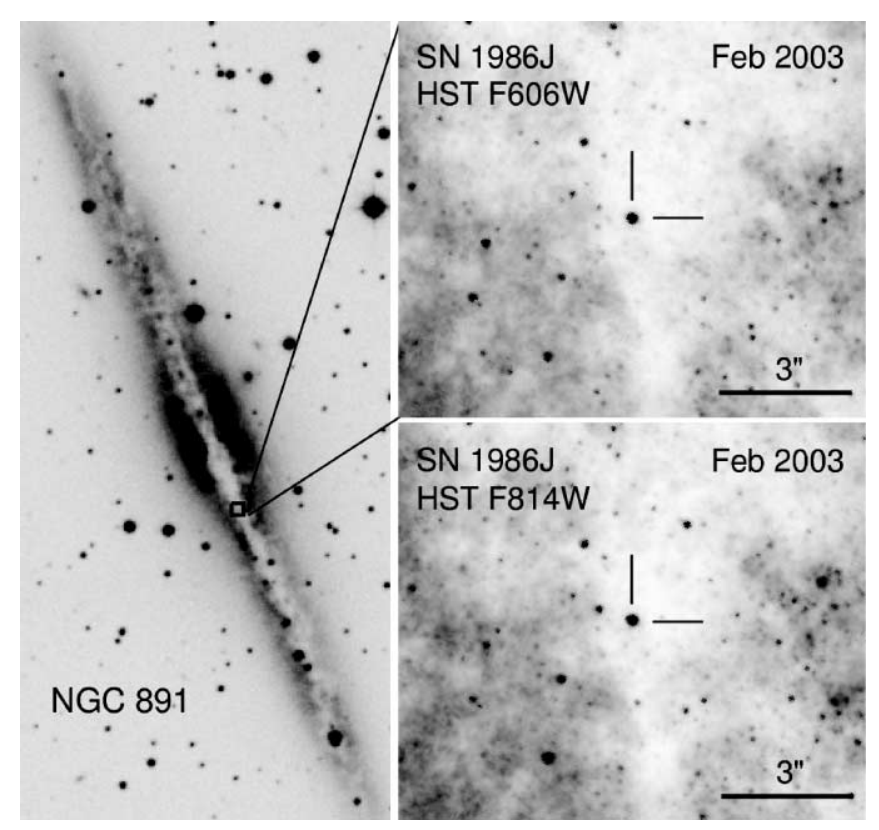

FIG. 1.-Left: DSS2 Blue image of NGC 891 with the location of SN 1986J marked. Right: 2003 February HST ACS/WFC F606W and F814W images of NGC 891 centered on SN 1986J. North is up and east is to the left.

panels show HST ACS/WFC images of NGC 891 centered on the region around the supernova. With 2003 epoch VEGAMAG apparent magnitudes of $m_{\mathrm{F} 606 \mathrm{~W}}=21.4$ and $m_{\mathrm{F} 814 \mathrm{~W}}=20.0 \mathrm{mag}$, these images indicate that SN $1986 \mathrm{~J}$ has remained relatively bright nearly two decades after the estimated 1983 optical outburst.

The SN 1986J site in NGC 891 was also imaged some 8 years earlier on 1994 December 1 by HST with the Wide Field Planetary Camera 2 (WFPC2) using the F606W filter (see van Dyk et al.
1999). Reduction of these 1994 data show $m_{\mathrm{F} 606 \mathrm{~W}}=21.3 \mathrm{mag}$. Accounting for differences in instrumental response between the WFPC2 and ACS, these observations suggest a decline of only $\lesssim 1 \mathrm{mag}$ in the F606W filter over the 8 years separating the observations.

\subsection{Emission Line Changes Since 1989}

In Figure 2 we present optical spectra of SN 1986J at three epochs spanning 18 years: 1989.7 (published by L91) 1991.8, and 2007.9. The 2007 spectrum is an average of the three spectra obtained in 2007 September and December, with the combined relative fluxes believed accurate to within $\pm 20 \%$.

Between the three epochs, SN 1986J's optical emission shows several significant changes. The greatest change is the decline in $\mathrm{H} \alpha$ emission. As of 2007, the $\mathrm{H} \alpha$ line observed centered around $6564 \AA$ has a flux of $4 \times 10^{-16} \mathrm{erg} \mathrm{s}^{-1} \mathrm{~cm}^{-2}$, down some 20 times from $9 \times 10^{-15} \mathrm{erg} \mathrm{s}^{-1} \mathrm{~cm}^{-2}$ observed in 1989 . The [ $\mathrm{N}$ II] $\lambda \lambda 6548,6583$ emission lines are not resolved, and we measure the $\mathrm{H} \alpha$ emission in 2007 assuming nitrogen contributes approximately $1 / 4$ of the total integrated flux around the line. We estimate that $\mathrm{H} \alpha$ emission declined by a factor of 2 between 1989 and 1991 and by a factor of 10 between 1991 and 2007.

The line center for SN 1986J's H $\alpha$ emission also showed an increasing blueshift between 1989 and 2007. In 1986, the line center roughly matched NGC 891's redshift of $+528 \mathrm{~km} \mathrm{~s}^{-1}$ (Rupen et al. 1987; de Vaucouleurs et al. 1991). However, by 1989 the shift had decreased to $+330 \mathrm{~km} \mathrm{~s}^{-1}$ (L91), and in 2007 the center of the observed $\mathrm{H} \alpha$ emission shifted still more to the blue, virtually negating the galaxy's systemic velocity and appearing practically unredshifted.

Other changes in the late-time spectra of SN 1986J include the fading beyond detectability of $\mathrm{H} \beta$ and $\mathrm{He}$ I $\lambda 5876$ and $\lambda 7065$ emission lines in the 2007 spectrum that were present in 1989 and 1991. Also considerably diminished in 2007 is the emission

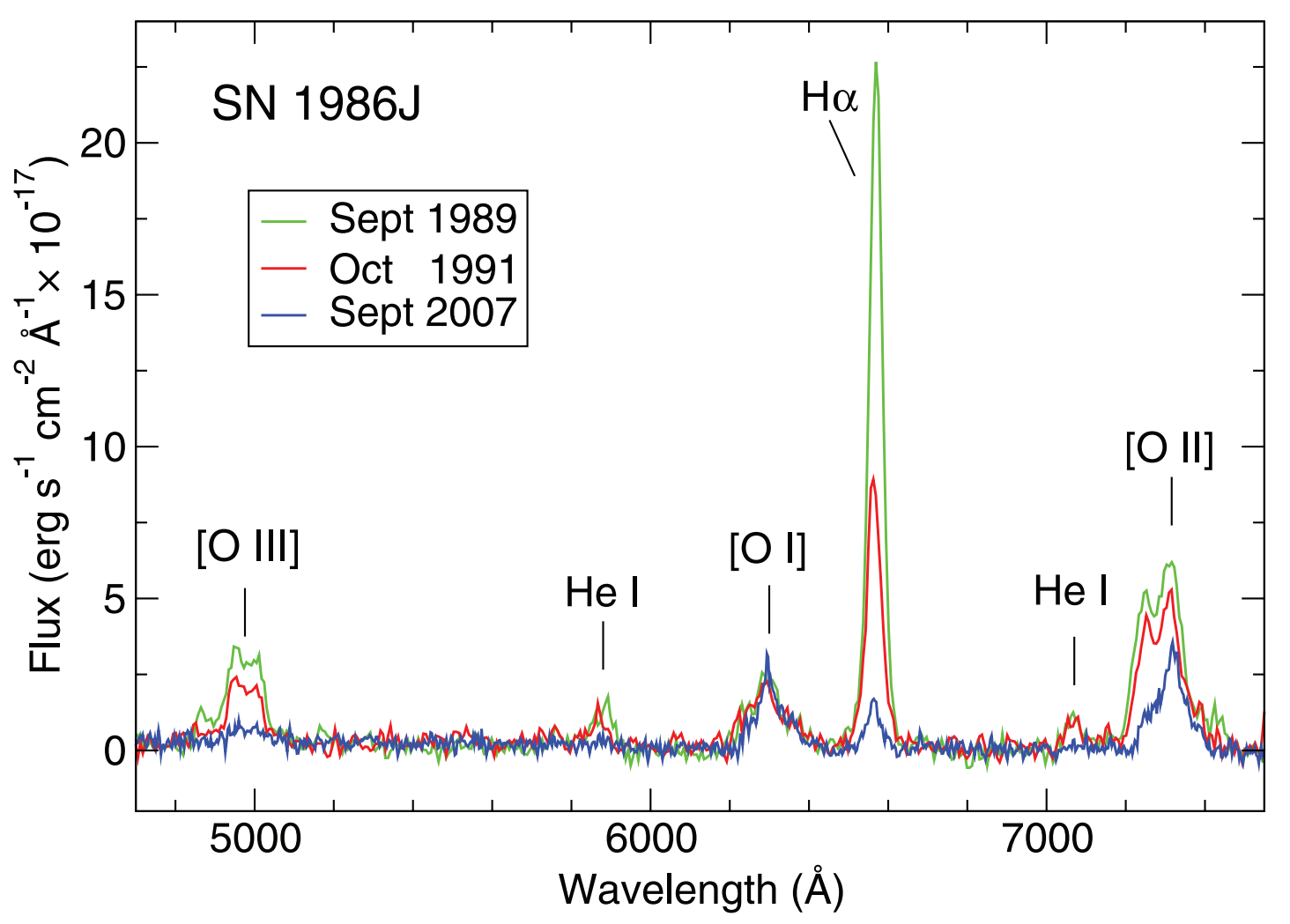

FIG. 2.-Optical spectra of SN 1986J at three epochs covering 18 years. 


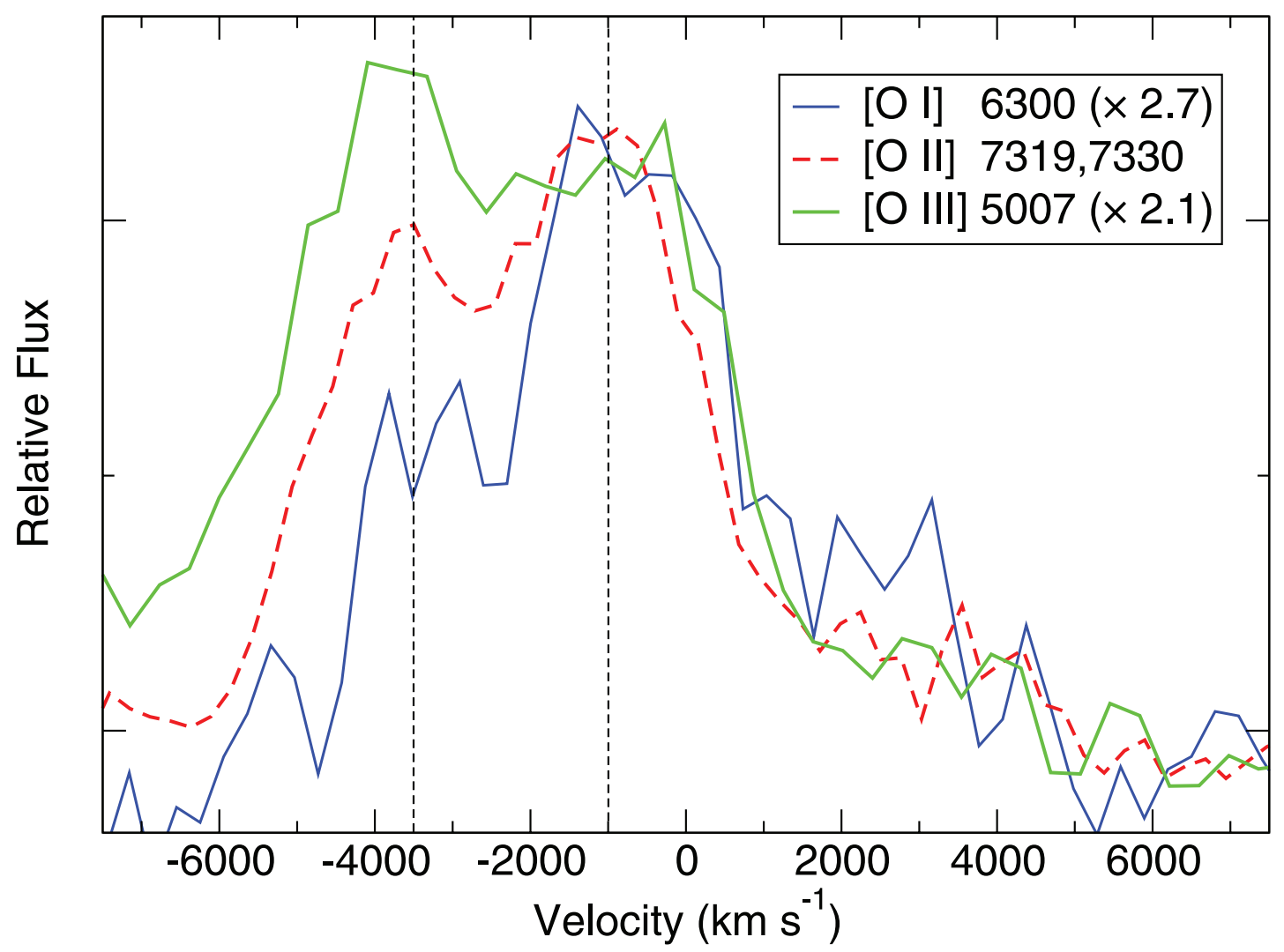

FIG. 3.-SN 1986J's 1989 oxygen line profiles. Velocities shown are with respect to 6300, 7325, and $5007 \AA$ in the rest frame of NGC 891 . Vertical dashed lines are positioned at $-1000 \mathrm{~km} \mathrm{~s}^{-1}$ and $-3500 \mathrm{~km} \mathrm{~s}^{-1}$.

associated with the $[\mathrm{O}$ III] $\lambda \lambda 4959,5007$ emission lines observed around $4980 \AA$.

Changes in the profiles of some emission lines are evident. Broad emission around $7300 \AA$, consisting of two prominent emission peaks at $\simeq 7250 \AA$ and $7320 \AA$, seen in both the 1989 and 1991 spectra exhibits a significant diminishment along its blue side in the 2007 spectrum (see Fig. 2). We identify both emission peaks with the $[\mathrm{O}$ II] $\lambda \lambda 7319,7330$ line emission. Although some contribution from [Ca II] $\lambda \lambda 7291,7324$ is possible, [O II] emission likely dominates the broad 7200-7350 A emission.

The bluer emission peak at $7250 \AA$, prominently visible in 1989 and 1991 spectra, faded significantly by 2007 , evolving into a weak, broad emission feature blueward of $7300 \AA$. The other emission peak at $7320 \AA$ showed a smaller intensity decline and a shift to the red by $\sim 10 \AA$ relative to its appearance in 1989 . Finally, faint redshifted [O II] emission extending from 7380 to $7450 \AA$ visible in the 1989 spectrum gradually weakened and decreased in velocity in the 1991 and 2007 spectra.

Other features show only minor changes in strength and/or profile. The broad emission centered around $6295 \AA$ identified with the [O I] $\lambda 6300$ line has a 2007.8 epoch flux of $1.4 \times 10^{-15} \mathrm{erg} \mathrm{s}^{-1}$ $\mathrm{cm}^{-2}$, assuming a ratio of $3: 1$ for the $6300 \AA$ and $6364 \AA$ lines. This is roughly the same as the combined flux of $2.7 \times 10^{-15} \mathrm{erg}$ $\mathrm{s}^{-1} \mathrm{~cm}^{-2}$ for the two lines measured in 1989 by L91.

\section{DISCUSSION}

\subsection{Emission-Line Profiles from O-Rich SN Ejecta}

While a blueshifted, double-peak emission profile is most apparent in the [O II] $\lambda \lambda 7319,7330$ lines in the 1989 and 1991 spectra, in fact, all of SN 1986J's oxygen emissions display a similar double-peak emission profile blueshifted with respect to the host galaxy's rest frame. Figure 3 presents an overlay of [O I], [O II], and [O $\mathrm{III}]$ line profiles plotted in velocity space. This figure shows good agreement for both the line profiles and emission peaks near -1000 and $-3500 \mathrm{~km} \mathrm{~s}^{-1}$.

Added support for double-peak oxygen line profiles comes from faint emission near $4340 \AA$ present in both 1989 and 1991 spectra, which we interpret as [O III] $\lambda 4363$ line emission (see Fig. 2 in L91). When corrected for NGC 891's redshift, the positions and widths of the peaks observed at $4313 \AA$ and $4338 \AA$ match the -1000 and $-3500 \mathrm{~km} \mathrm{~s}^{-1}$ emission peaks observed in the other oxygen profiles. After correcting for foreground reddening of $A_{V}=1.5 \mathrm{mag}$ (Rupen et al. 1987), the observed [O III] $I(4959+5007) / I(4363)$ line ratio $\simeq 2$ suggests an electron density for the $[\mathrm{O} \mathrm{III}]$ emitting region of $(3-5) \times 10^{6} \mathrm{~cm}^{-3}$, assuming an electron temperature of $(2.5-5.0) \times 10^{4} \mathrm{~K}$, like that found in shock-heated O-rich ejecta seen in young supernova remnants (Hurford \& Fesen 1996; Blair et al. 2000).

The interpretation of a spectrum dominated by two blueshifted, O-rich ejecta clumps is very different from that proposed by L91 to explain the boxlike [O III] $\lambda \lambda 4959,5007$ profile. They suggested that the observed shape was due to the $\lambda 5007 / \lambda 4959$ line ratio being close to $1: 1$ instead of the optically thin ratio of $3: 1$ typically observed in low-density nebulae. A ratio close to unity for both the [O I] and [O III] line doublets was interpreted as caused by emission originating from regions with electron densities of $n_{e} \sim 10^{9} \mathrm{~cm}^{-3}$. However, in light of the strong similarity of all oxygen emission profiles, such high density estimates no longer appear to be required.

\subsection{Origin of the Late-Time Optical Emission}

Our interpretation of line emission profiles together with the observed spectral evolution over the last two decades suggests 
two separate sites for SN 1986J's late-time optical emission. The decline of SN 1986J's H $\alpha$ emission and its relatively low expansion velocity $\left(<700 \mathrm{~km} \mathrm{~s}^{-1}\right)$ suggests that this emission comes from shock-heated circumstellar material (CSM). Early spectra showing an initially very bright $\mathrm{H} \alpha$ emission along with fainter emissions from $\mathrm{He}$ I and [ $\mathrm{Fe}$ II] are consistent with an emission nebula generated by a $\sim 1.5 \times 10^{4} \mathrm{~km} \mathrm{~s}^{-1}$ blast wave overrunning a dense CSM environment rich in CNO-processed material (Rupen et al. 1987). The apparent blueshift in the line center of $\mathrm{H} \alpha$ over the past 20+ years is likely due to increasing extinction of the receding hemisphere, possibly due to dust formation in the SN ejecta.

Chugai \& Danziger (1994) suggested that SN 1986J's latetime optical emission originates from shocked dense clouds of circumstellar gas in the progenitor star's clumpy pre-SN wind. The presence of [N $\mathrm{NI}] \lambda 5755$ line emission and the lack of strong [N II] $\lambda \lambda 6548,6583$ emission (Rupen et al. 1987) suggest relatively high densities $\left(n_{e} \sim 10^{6} \mathrm{~cm}^{-3}\right)$, similar to those seen in the circumstellar ring around SN 1987A.

The interaction of the SN's outward-moving blast wave with dense and clumpy CSM will generate a strong reverse-shock into slower expanding SN ejecta, leading to the observed forbidden oxygen line emissions. The presence of two prominent blueshifted emission peaks across three ionization stages implies that this component of SN 1986J's optical emission comes mainly from two large patches of reverse shock-heated, O-rich ejecta on the facing expanding hemisphere having radial velocity components in our line of sight around -1000 and $-3500 \mathrm{~km} \mathrm{~s}^{-1}$.

The gradual redward shift of the $-1000 \mathrm{~km} \mathrm{~s}^{-1}$ emission component toward smaller blueshifted velocities, seen most clearly in the $\left[\mathrm{O}_{\text {II }}\right] \lambda \lambda 7319,7330$ profile between 1989 and 2007, may signal the progression of reverse shock emission coming from inner, slower moving O-rich ejecta during the intervening two decades. In addition, weak emission seen redward of $7330 \AA$, together with weak emission near $5050 \AA$, possibly associated with [O III], might indicate highly reddened O-rich ejecta located on the rear expanding hemisphere with radial velocities up to $3500 \mathrm{~km} \mathrm{~s}^{-1}$.

Finally, we should mention the possibility of photoionization of SN 1986J's ejecta by its bright central compact source (Chevalier 1987). Early optical and radio observations of SN 1986J suggested that it may be a very young Crab Nebula-like rem- nant (Chevalier 1987; Weiler et al. 1990), and such a connection has been strengthened by recent VLBI observations showing a bright, compact radio component with an inverted spectrum near the center of the expanding shell (Bietenholz et al. 2004; Bietenholz \& Bartel 2008). This central source is thought to be associated with either a young, energetic neutron star or accretion onto a black hole. The optical filaments in the Crab Nebula are mainly photoionized by its pulsar's synchrotron nebula, and with SN 1986J's central component some 200 times more luminous than the Crab Nebula between 14 and $43 \mathrm{GHz}$ (Bietenholz $\&$ Bartel 2008), this raises the possibility of photoionization of SN 1986J's ejecta.

During its first decade of evolution, however, SN 1986J's strong [O III] $\lambda 4363$ line suggested temperatures more indicative of shock heating $(T \gtrsim 25000 \mathrm{~K}$ ) rather than photoionization $(T \leq 15000 \mathrm{~K})$. In addition, the large Balmer decrement ratio of $\mathrm{H} \alpha / \mathrm{H} \beta \sim 45$ (1986.8 epoch; L91) observed in early spectra pointed to dense, shocked gas. High densities in O-rich ejecta and/or the formation of dust in the ejecta would limit the importance of photoionization by the central source at early times.

On the other hand, at its current age of $\sim 25 \mathrm{yr}$, the importance of photoionization from the central source, quite possibly a young Crab-like neutron star, is less clear. Recent observations with XMM-Newton and Chandra have shown a sharp decline in X-ray luminosity, perhaps signaling a diminishing role of shock-heating in SN 1986J's late-time optical emission (Temple et al. 2005). Future increased contributions from photoionization could be reflected as a broadening in optical emission line widths, like that predicted by Chevalier \& Fransson (1992).

In view of SN 1986J's strong oxygen line emissions, a better comparison than the Crab might be the $\simeq 1000 \mathrm{yr}$ old LMC remnant 0540-69.3. This remnant has a bright pulsar wind nebula surrounding a $50 \mathrm{~ms}$ pulsar and shock-heated, O-rich ejecta expanding at velocities $\sim 2000 \mathrm{~km} \mathrm{~s}^{-1}$ (Morse et al. 2006), much more in line with what is observed in SN 1986J.

We thank R. Chevalier and M. Bietenholz for helpful comments on an earlier draft. This research was supported in part by a Canadian NSERC award to D. M.
Bartel, N., Rupen, M. P., Shapiro, I. I., Preston, R. A., \& Rius, A. 1991, Nature, 350,212

Bartel, N., Shapiro, I. I., \& Rupen, M. P. 1989, ApJ, 337, L85

Bietenholz, M. F., \& Bartel, N. 2008, Adv. Space Res., 41, 424

Bietenholz, M. F., Bartel, N., \& Rupen, M. P. 2002, ApJ, 581, 1132 2004, Science, 304, 1947

Blair, W. P., et al. 2000, ApJ, 537, 667

Chevalier, R. A. 1987, Nature, 329, 611

Chevalier, R. A., \& Fransson, C. 1992, ApJ, 395, 540

Chugai, N. N., \& Danziger, I. J. 1994, MNRAS, 268, 173

de Vaucouleurs, G., de Vaucouleurs, A., Corwin, H. G., Jr., Buta, R. J., Paturel, G.,

\& Fouque, P. 1991, Third Reference Catalogue of Bright Galaxies, Vol. 9 (Berlin: Springer)

Hurford, A. P., \& Fesen, R. A. 1996, ApJ, 469, 246

\section{REFERENCES}

Kent, S., \& Schild, R. 1987, IAU Circ., 4423, 1

Leibundgut, B., et al. 1991, ApJ, 372, 531 (L91)

Morse, J. A., Smith, N., Blair, W. P., Kirshner, R. P., Winkler, P. F., \& Hughes, J. P. 2006, ApJ, 644, 188

Rupen, M. P., van Gorkom, J. H., Knapp, G. R., Gunn, J. E., \& Schneider, D. P. 1987, AJ, 94, 61

Schlegel, E. 1990, MNRAS, 244, 269

Strom, K. M. 1977, Kitt Peak National Observatory Memorandum, Standard Stars for Intensified Image Dissector Scanner Observations (Tucson: KPNO) Temple, R. F., Raychaudhury, S., \& Stevens, I. R. 2005, MNRAS, 362, 581 van Dyk, S. D., Peng, C. Y., Barth, A. J., \& Filippenko, A. V. 1999, AJ, 118, 2331 van Gorkom, J., Rupen, M., Knapp, G., Gunn, J., Neugenbauer, G., \& Matthews, K. 1986, IAU Circ., 4248, 1

Weiler, K. W., Panagia, N., \& Sramek, R. A. 1990, ApJ, 364, 611 